\title{
Exploration on Ability Training and Practical Training of English Interpretation
}

\author{
Yanmei Jia \\ College of Foreign Languages, Leshan Normal University, Leshan, 614000, China
}

Keywords: English interpretation, Ability training, Practical training

\begin{abstract}
With the constant acceleration of Chinese reform process, China has increasingly frequent association with various countries in the world in economy, politics and culture and social demands for interpretation talents increase year by year. To improve interpretation ability of college students, colleges and universities respectively explore different interpretation training modes. Though corresponding progress has been made, it cannot meet current social demands and students' needs. Therefore, the improvement of college English interpretation teaching quality and students' interpretation ability and the delivery of professional interpretation talents to the society become the priority among priorities in interpretation teaching of colleges and universities.
\end{abstract}

\section{Introduction}

Interpretation is a form of oral expression, i.e. accurate and fast expression of the language heard with another language for the purpose of realizing information transmission and exchange. It is a most basic linguistic communication method of human beings in cross-cultural communication. ${ }^{[1]}$ This paper conducts in-depth research on interpretation ability training according to features and problems of college English interpretation and explores feasible methods so as to make contributions to Chinese interpretation cause.

\section{Interpretation overview}

\section{Features of interpretation}

Unpredictability

Interpretation is an exchange activity between two languages with unpredictability. Generally, formal occasions such as top-level conference or international press conference require interpretation. It is a test for both the professional quality and psychological quality of interpreters. Meanwhile, these occasions are on-site, instant and changeful. Therefore, both the content and form of interpretation are unpredictable.

Field pressure

Another feature of interpretation work is that field pressure exists in the work process. The interpretation site is sometimes very serious, e.g. important occasions such as international conference. Serious conference atmosphere will undoubtedly impose pressure on the psychology of interpreters. Some persons with inadequate experience even have error in speaking. Therefore, interpretation requires good psychological quality of workers.

Individual operability

Interpretation workers require a strong ability of independent operation. Therefore, workers should have the ability of handling emergency situations at any time in the whole interpretation process.

Comprehensive application of listening, speaking, reading and writing

Interpretation is a comprehensive activity with comprehensive application of listening, speaking, reading and writing abilities. ${ }^{[2]}$ Accurate interpretation of a sentence not only requires strong oral competence, but also is a comprehensive manifestation of translation, dictation and thinking abilities. Therefore, it has very high requirements for comprehensive language competence of interpreters. 


\section{Rich contents}

Interpreting communication has very rich contents. This is another major feature of interpretation. Interpretation is a job with extremely strong specialty, which requires strong specialty foundation, high psychological quality and ability of fluent expression of interpretation workers.

\section{Types of interpretation}

Interpretation can be divided into consecutive interpretation and simultaneous interpretation according to operation form of interpretation.

1). Consecutive interpretation: a single interpretation way with listening prior to interpretation in which a sentence or paragraph is the unit. Consecutive interpretation applies to multiple occasions such as lecture, lesson and press conference.

2). Simultaneous interpretation: a translation method of simultaneous listening and interpretation. Workers interpret the content of speech ceaselessly without interrupting the speaker and meanwhile deliver the content to audiences. The greatest advantage of this interpretation method is high efficiency. It is the most common and basic interpretation method in international conferences. Simultaneous interpretation is also applied in such occasions as academic report and lecture.

Interpretation can be divided into tour interpretation, conference interpretation, information interpretation and ceremony interpretation according to content.

\section{Existing problems of current interpretation training}

Due to the influence of traditional educational form, modern interpretation course reform has started late and interpretation teaching has various problems. In daily interpretation training, students mainly have the following problems:

First, emphasize interpretation but neglect translation. This is the most common phenomenon in interpretation teaching training. ${ }^{[3]}$ The ability of fast Chinese-English transformation is a major advantage of professional interpreters. However, emphasizing interpretation excessively and neglecting translation will make them have such problems as simple sentence pattern and rigid semantics in the interpretation process. The function of translation is to exercise linguistic embellishment ability of interpreters and allow them to interpret required contents expertly and vividly in the interpretation process.

Second, emphasize translation but neglect interpretation. This is a common phenomenon in interpretation teaching. Translation training can help interpreters accumulate knowledge rapidly in such aspects as morphology, syntax and grammar. If interpreters only accumulate knowledge without output, it is adverse to the training of interpretation ability.

The training in these two aspects is indispensable for excellent interpretation talents. Neglecting either aspect is adverse to the training of interpretation ability.

\section{Skill training in interpretation teaching}

Interpretation teaching is a professional discipline integrating the learning of theoretical knowledge and skill training. Therefore, it should follow the teaching principle combining theory and practice, i.e. with the learning of theoretical knowledge as base in combination with reasonable training method. The objective of interpretation teaching is to train students' interpretation ability. The training of skills is often completed in training form. The training of these skills includes training of listening skills, reading comprehension skills, memory skills, linguistic skills and interpretation skills.

\section{Listening skill training}

There are two basic standards for judging the quality of interpretation: accuracy and fluency. Accuracy is the foundation of interpretation. The basic process of interpretation includes information input, information interpretation and information output. ${ }^{[4]}$ Accurate information input is the foundation of the whole interpretation process and plays a decisive role in the interpretation process. 
Listening is the first step of interpretation. Therefore, interpretation has high requirements for listening. Interpreters should not only hear each word clearly, but also understand the purpose and tone of the speaker and the deep meaning of words. Generally, there are many contents of listening which are very professional because interpretation occasions are mostly senior conference or press conference. This requires interpretation workers to focus attention, have the ability of short-term memory and be familiar with different English accents, voices of different tones and different speaking habits.

To achieve the purpose of specialization, super-strength listening training is required. However, the main method of listening teaching is classroom teaching. The amount of listening is far from enough. Students' listening training is a basic skill. This requires long-term, extensive and delicate training, because listening training should accompany interpretation teaching.

\section{Reading skill training}

Reading comprehension is the second major item of English interpretation as well as a vital link in English skill training. Reading comprehension occupies a large space in domestic English tests and various large international English tests. Therefore, the training of reading comprehension is very important. It lays a solid foundation for the training of interpretation skills. Reading is not strange for us. It mainly includes main idea questions and detail questions. High-efficiency reading comprehension skills can help us grasp the main idea of an article accurately, understand the gist of a paragraph and narrative thinking of the article and answer questions. How to master skills for answering questions? We should know the following:

Pay attention to the beginning and the end and master the gist of the article

In English reading, the first and last paragraphs of an article describe the gist of the article or opinions of the author. The first and last sentences in a paragraph often show the theme of the article. Therefore, it is required to pay attention to the beginning and the end of article, which can help us master the gist of the article.

Pay attention to the turning point of the article

The turning point of the article is often the main point of topic. Mastering the turning point means mastering the bone and vein of the article. It will greatly save our time for answering questions.

Pay attention to question stem and eye

After grasping question stem and eye, we can determine the position of a question in the article rapidly ${ }^{[5]}$ and find the answer.

Pay attention to lexical meaning guess

We often meet strange words in an article. We cannot look up such words in the dictionary immediately. The omission of such words will influence our understanding of the whole article. Therefore, we need to guess lexical meaning. Lexical meaning should be guessed according to certain context. If the keynote is positive, the vocabulary might have a positive meaning. On the contrary, negative context makes words have negative meaning. Lexical meaning guess based on context is a basic method of reading. We need to master this method skillfully.

\section{Memory training}

The memory of human brain includes short-term memory and long-term memory. The former is an immediate memory with very limited amount of information and short retention time. The latter is a purposeful permanent memory which requires in-depth understanding and constant memorization of information. Interpretation teaching requires special training according to teaching contents.

Shadowing practice

Shadowing practice is retelling exercise in the same language, i.e. restatement of contents to be translated in the same language. This method can exercise the attention and understanding of interpretation practitioners. At the beginning of training, students can narrate synchronously with the recording. After a period of time, students can retell the content after the recording begins. 
Overview in the same language

This step continues the step above. Students can summarize the original text with memory and the core thought of the content after the end of repeat in the same language. This step aims at exercising students' ability of short-term memory and training students' habit of speaking while listening.

Overview in the target language

Overview in the target language can be exercised after the practice of overview in the same language. That is to say, the original text is summarized in the target language. During the practice, students do not need to pay excessive attention to sentence structure and contents. They only need to deliver the core thought and main information points to be expressed by the speaker accurately.

\section{Linguistic skill training}

Interpretation is a comprehensive linguistic activity including knowledge and skills such as listening, speaking, reading and writing. It is characterized by unpredictability, rich interpretation contents, great pressure and strong individual operability. This requires interpreters to have solid language foundation. An excellent interpreter should not only master the basic knowledge of language, but also have the ability of applying linguistic knowledge skillfully. He should not only be a person quick on the trigger and brilliant, but also have good abilities of memory and adaptability to changes.

\section{Interpretation of figures}

Different means of expression of English and Chinese in figures make the interpretation of figures very difficult. Chinese figures are expressed with the multiple of "ten", while English figures are expressed with the multiple of "thousand". This requires interpreters to have the ability of rapid transformation during the interpretation of figures. The practice of interpretation of figures can mainly involve restatement of figures with increasing amount and difficulty. This can strengthen both figure practice and memory ability.

\section{Adaptability to changes}

As interpretation has unpredictability, interpreters should have the ability of adapting themselves to changing circumstances. When meeting difficult vocabularies such as proverbs and allegorical saying, workers without experience often interpret them mechanically, thus resulting in inaccurate contents of translation. In addition, some new words in Chinese have emerged with the economic development and the popularization of network. These vocabularies do not have corresponding translation. This requires interpreters to have certain adaptability to changes during interpretation. Therefore, English interpretation teaching should pay attention to the training of students' adaptability to changes besides constant accumulation of knowledge. For example, teachers can set up simulated conference scenes or provide students with a lot of opportunities for practice. These are good methods for improving students’ adaptability to changes.

\section{Interpretation skill training}

Interpretation is a strongly professional communicative activity. Therefore, the following should be realized during training:

Develop good habits

Good habits are the foundation of success. Interpretation training should involve more listening and writing. It is required to follow the information during interpretation and not to remain on the previous information of the speaker or recall the information not heard clearly. Meanwhile, good psychological quality should be developed. Too nervous state should be avoided because only with peaceful mind can the optimal state be achieved.

\section{Practice makes perfect}

Interpretation generally has three processes: first, practice "feel". This process aims at training students' abilities of comprehension and fast reaction. Therefore, materials can be selected widely and involve as many fields as possible in this stage. Second, specific interpretation training. In this process, live recording of news and conferences can be selected for actual interpretation training. Third, practical training. Such training can be realized through interpretation test and internship. 


\section{Expand background knowledge}

Mastering the background knowledge of information can help interpreters translate information contents. Even facing contents not understood, interpreters can translate some contents according to the background knowledge of information. Therefore, this will undoubtedly help students in interpretation.

\section{Conclusion}

Interpretation is a communicative behavior of transforming the information from one language to another in oral form. It is not only theoretical knowledge, but also a skill. Therefore, it can be fully mastered only through a lot of practice and exercise. Students can become qualified interpretation talents only by promoting the spirit of perseverance and paying attention to the use of interpretation methods.

\section{References}

[1] Zhao Liang. English Listening and Interpretation - Brief Discussions on Improvement of Listening Ability in English Interpretation Training. Northern Literature: II, 2012(1):120-121.

[2] Li Mengzhuo. Brief Analysis on Short-term Memory Training for Improving English Interpretation Quality. Kaneda, 2013(9):186.

[3] Lv Sisheng. Test Report on Influence of "Loading Over-speed Interpretation Training Method” on English Interpretation. Hebei Normal University, 2013.

[4] Liu Qingxue. Research on Multi-modal Teaching Model of English Interpretation Course. Leading Edge, 2012(10):160-161.

[5] Wan Lihua, Tian Chuan. Listen Prior to Interpretation - First Exploration on Classroom Teaching Reform of English Interpretation. Art Science and Technology, 2013,26(4):139,131,136. 